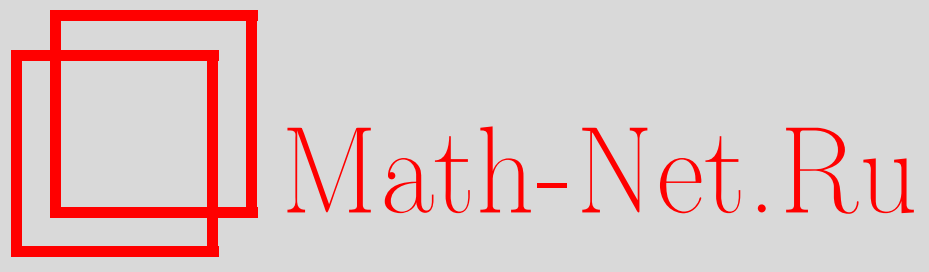

В. П. Маслов, О минимизации операционных рисков, Матем. заметки, 2006, том 80, выпуск 4, 569-572

DOI: https://doi.org/10.4213/mzm2849

Использование Общероссийского математического портала Math-Net.Ru подразумевает, что вы прочитали и согласны с пользовательским соглашением http://www . mathnet.ru/rus/agreement

Параметры загрузки:

IP: 35.173 .219 .12

26 апреля 2023 г., 15:16:42






\section{О МИНИМИЗАЦИИ ОПЕРАЦИОННЫХ РИСКОВ}

\section{В. П. Маслов}

Приводится формула для государственных вложений, минимизирующая риск. При этом учитывается закон "нулевого интеллекта" финансовых рынков.

Библиография: 3 названия.

Заместитель председателя Центрального Банка России Т. В. Парамонова поставила мне актуальную задачу о снижении операционных рисков. Операционные риски связаны, с одной стороны, со случайными происшествиями в системе управления инвестиционными вкладами государственных структур, подобных ЦБ, и, с другой стороны, с рисками самих вложений, определяемыми финансовыми рынками. Отметим, что разложение вкладов государственных структур по банкам, фирмам или финансовым инструментам не носит статистического характера, а является индивидуальным набором (последовательностью) и, следовательно, не относится к математической статистике и теории вероятностей. Эта задача ближе к теории колмогоровской сложности.

Специфика задачи состоит в том, что предприятия, в которые государство хочет сделать вложения, задаются заранее. Возможно, из политических соображений, или соображений доходности, или же из общих экономических стратегий заранее задаются предприятия $A_{1}, A_{2}, \ldots, A_{s}$, где $s$ достаточно велико. Кроме того, государство устанавливает порядок приоритетов среди этих предприятий: предприятие $A_{i}$ более перспективно, чем предприятие $A_{i-1}$. Это означает, что акций (облигаций) у предприятия $A_{i-1}$ нужно купить не больше, чем у $A_{i}$. В остальном же нужно разложить деньги (купить акции) по этим предприятиям так, чтобы операционный риск был минимальным. Государственный орган указывает, сколько вложить в наиболее приоритетное предприятие $A_{s}$ и в наименее приоритетное предприятие $A_{1}$, а также бюджетное ограничение.

В решении этой задачи мы будем придерживаться концепции “отсутствия интеллекта" (zero intelligence) для финансовых рынков. Эта концепция возникла при исследовании Лондонской фондовой биржи. Этот феномен обсуждается в постоянно возрастающем потоке публикаций. Однако с нашей точки зрения [1] этот эффект вполне естественен.

Работа выполнена при поддержке Российского фонда фундаментальных исследований, грант № 05-01-00824. 
Эффект заключается в том, что трейдеры по данным биржи случайным образом ("не прибегая" к интеллекту) составляют свои портфели. Однако согласно концепции Колмогорова случайность - это большая сложность. Так, если мы будем случайным образом делать ставки на орла или решку, или же построим длинный алгоритм и будем его придерживаться, все равно примерно в половине случаев выпадет орел, а в половине - решка.

Продолжим это сравнение. Мы кидаем монетку $S$ раз, где $S$ велико, и при этом повторяем $S$ бросаний еще $N$ раз. Все варианты выпадения, в том числе одних орлов или попеременного выпадения орлов и решек, равновероятны (и очень мало вероятны). Но большинство из вариантов будет сходиться к примерно равному числу орлов и решек. Это означает, в частности, что если мы будем играть в орла и решку и ставить в половине случаев на орла, а в половине на решку, то риск будет минимальный, но и выигрыш тоже.

Поэтому если мы найдем наибольшее "скопление" вариантов для нашей задачи, которые равновероятны в силу принципа "отсутствия интеллекта", то именно такое разложение будет наименее опасное, безрисковое.

Пусть согласно условию $C_{i}$ - число акций, приобретенных государством у $i$-го предприятия (банка), - удовлетворяет соотношению

$$
C_{i+1} \geqslant C_{i}, \quad i=1,2, \ldots, s, \quad C_{s} \leqslant M, \quad C_{1} \geqslant K,
$$

причем $M$ и $K$ заданы. Отсюда

$$
C_{s}-C_{1} \leqslant M-K
$$

Пусть $p_{i}$ - цена акции $i$-го предприятия. Очевидно, что

$$
\sum_{i=1}^{s} C_{i} p_{i} \leqslant C_{s} \sum_{i=1}^{s} p_{i}
$$

Поэтому бюджетное ограничение $\Phi$ удовлетворяет соотношению

$$
C_{1} \sum_{i=1}^{s} p_{i} \leqslant \sum_{i=1}^{s} C_{i} p_{i} \leqslant \Phi \leqslant C_{s} \sum_{i=1}^{s} p_{i},
$$

т.е. при этом естественном неравенстве для бюджетного ограничения $\Phi$

$$
\sum_{i=1}^{s} C_{i} p_{i} \leqslant \Phi
$$

Имеем

$$
\sum_{i=1}^{s} C_{i} p_{i} \equiv \sum_{i=2}^{s}\left(C_{i}-C_{i-1}\right) \sum_{j=i}^{s} p_{j}+C_{1} \sum_{i=1}^{s} p_{i} \leqslant \Phi,
$$

откуда

$$
\sum_{i=2}^{s}\left(C_{i}-C_{i-1}\right) \sum_{j=i}^{s} p_{j} \leqslant \Phi-C_{1} \sum_{i=1}^{s} p_{i}
$$

Обозначим

$$
n_{i}=C_{i}-C_{i-1}, \quad \lambda_{i}=\sum_{j=i}^{s} p_{j}
$$


Отсюда

$$
\sum_{j=2}^{i} n_{j}=C_{i}-C_{1}, \quad \lambda_{i} \leqslant \lambda_{i-1} \leqslant \cdots \leqslant \lambda_{1}, \quad \lambda_{1}=\sum_{j=1}^{s} p_{s} .
$$

Мы получаем

$$
\sum_{i=2}^{s} n_{i} \lambda_{i} \leqslant \Phi-K \sum_{j=1}^{s} p_{s}
$$

Обозначим $\Phi^{\prime}=\Phi-\sum_{i=1}^{s} p_{i} C_{1}$.

Рассмотрим множество всех $\left\{n_{i}\right\}$ таких, что

$$
\sum_{i=2}^{s} n_{i} \lambda_{i} \leqslant \Phi^{\prime}
$$

Поскольку набор $\left\{C_{i}\right\}$ равновероятен при условиях (1) и $(2)$, отсюда следует, что $n_{i}=C_{i}-C_{i-1}, i=2, \ldots, s$, удовлетворяющие равенству

$$
\sum_{i=2}^{s} n_{i} \leqslant M-K
$$

равновероятны при условии $n_{i} \geqslant 0$ и условии (9).

Теорема 1. Пусть $R=\min (s, M-K)$. Доля наборов $\left\{n_{i}\right\}, i=2, \ldots, s$, таких, что выполнено (9), и для которых

$$
\left|C_{i}-C_{1}-\sum_{j=2}^{i} \frac{1}{e^{\beta \lambda_{j}-\sigma}-1}\right| \geqslant R^{1 / 2+\varepsilon},
$$

меньше, чем $R^{-d}$, где d сколь угодно велико, $\sigma$ - константа, $i>\varepsilon R, \varepsilon$ сколь угодно мало.

Константы $\sigma$ и $\beta$ находятся из условий

$$
\begin{gathered}
\sum_{j=2}^{s} \frac{1}{e^{\beta \lambda_{j}-\sigma}-1}=M-K, \\
\sum_{j=2}^{s} \frac{\lambda_{j}}{e^{\beta \lambda_{j}-\sigma}-1}=\Phi^{\prime} .
\end{gathered}
$$

Теорема 1 доказывается в работах [2] и [3].

СлЕДСТВИЕ. Безрисковое вложение (покупка акций) при условиях (1)-(3) осуществляется, если

$$
C_{i}=C_{1}+\sum_{j=2}^{i} \frac{1}{e^{\beta \sum_{k=j}^{s} p_{k}-\sigma}-1}, \quad i>\varepsilon R,
$$

где $\sigma$ и $\beta$ определяются из условий

$$
\sum_{j=2}^{s} \frac{1}{e^{\beta \sum_{k=j}^{s} p_{k}-\sigma}-1}=M-K, \quad \sum_{i=2}^{s} p_{i} \sum_{j=2}^{i} \frac{1}{e^{\beta \sum_{k=j}^{s} p_{k}-\sigma}-1}=\Phi-p_{1} K .
$$




\section{СПИСОК ЦИТИРОВАННОЙ ЛИТЕРАТУРЫ}

[1] В. П. Маслов, "О принципе возрастания сложности формировании портфеля на фондовой бирже", Докл. РАН, 404:4 (2005), 446-450.

[2] В. П. Маслов, "Закон "отсутствия предпочтения" и соответствующие распределения в частотной теории вероятностей", Матем. заметки, 80:2 (2006), 220-230.

[3] V.P. Maslov, "The Zipf-Mandelbrot law: quantization and an application to the stock market", Russ. J. Math. Phys., 12:4 (2005), 483-488.

В. П. Маслов

Поступило

Московский государственный университет

18.08 .2006

им. М. В. Ломоносова

E-mail:v.p.maslov@mail.ru 